\title{
ARCAT: UN PROGRAMA INFORMÁTICO PARA EL ANÁLISIS DEL ARTE ROMÁNICO CATALÁN
}

\author{
M. TERESA MATAS I BLANXART \\ Institut d'Estudis Catalans
}

\begin{abstract}
SUMARIO
I. Presentación.- II. Objetivos.- III. Estructura de la base de datos ARCAT: 1. Ficha técnica.- 2. Grupos de documentación.- 3. Variables.IV. Sistemas de consulta.- V. Protocolos de conexión y consulta.
\end{abstract}

\section{PRESENTACIÓN}

La idea de llevar a cabo un programa informático dedicado al análisis del arte románico catalán, surge como consecuencia de la creación en la sede del Institut d'Estudis Catalans, a lo largo del año 1984, del Centre d'Estudis i Desenvolupaments Informàtics (CEDI).

En el año 1985, la Sección Histórico-Arqueológica de la citada entidad, aceptó el estudio de los edificios románicos catalanes como un trabajo de investigación integrado en dicha sección, siendo reconocido con el nombre de Base de Dades d'Art Romànic Català (ARCAT). Este proyecto, fue avalado por los doctores Anscari M. Mundó y Joan Ainaud de Lasarte, y dirigido por el Dr. Joan-F. Cabestany i Fort y la Dra. M. Teresa

"Anuario de Estudios Medievales", 26 (1996) 
Matas i Blanxart. Los primeros resultados de la creación de esta base de datos se dieron a conocer el 20 de octubre de $1986^{1}$.

En el transcurso del año 1988, la Fundación Paul Getty de EEUU y concretamente a través del programa The Getty Art History Information Program, reconoció el interés científico de ARCAT, incorporándolo y haciéndolo figurar entre los proyectos del SN/G Report on Data Processing Projects in Art de la Getty Art History Information Program ${ }^{2}$.

ARCAT pasó a formar parte de los programas de investigación del Institut d'Estudis Catalans a petición del Dr. Joan Ainaud de Lasarte (Miembro de la Sección Histórico-Arqueológica) y del entonces vicepresidente del IEC, Dr. Enric Casassas. A partir de 1990, se modifica su nombre pasandose a denominar Centre d'Art Romànic Català (ARCAT). En el mes de octubre del mismo año se organizó, bajo el soporte de la Comissió Interdepartamental de Recerca i Innnovació Tecnológica (CIRIT) y el Institut d'Estudis Catalans, un seminario dedicado a La problemática del estudio de las obras de arte a través de sistemas informáticos. Este fue impartido por la profesora Cecilia Parra de la Scuola Normale Superiore de Pisa, designada por el profesor Dr. Salvatore Settis, director de la citada escuela, quien conoció el interés del programa ARCAT a través del director de la Fundación Paul Getty de Los Angeles. Participó también en dichas jornadas Mme. Catherine Arminjon de la Direction du Patrimoine del Ministère de la Culture et de la Comunication (Paris) ${ }^{3}$.

\footnotetext{
'Tras la presentación pública, y como consecuencia de la aceptación obtenida por la Base de Dades d'Art Romànic Català (ARCAT), fueron publicados algunos artículos, en prensa y revistas especializadas, entre los cuales destacan: M. Teresa MATAS I BLANXART, E. CARDONA I MIRET, Creació, criteris, metodologia i problemàtica del Programa d'Anàlisi d'Art Romànic Català (ARCAT), "Lambard. Estudis d'Art Medieval", III (1987), pp. 1-52. Lluis BonADA, El Programa d'Anàlisi ARCAT una tasca de l'Institut d'Estudis Catalans, "Diari Avui", (9 de diciembre de 1986). M. Teresa MATAS I BlanXarT. Esperança PIQUER I FerRer, ARCAT; un arxiu d'Art Romànic de Catalunya, "L'Avenç", 15 (1988), pp. 249-251. M. Teresa MATAS BLANXART, Un Banc de Dades Informatitzat per l'Art Románic Català, "La Vanguardia" (31 de enero de 1989). Carles SingLA, L'IEC recollirà en una Base de Dades les imatges dels edificis romànics, "Diari de Barcelona" (27 de mayo de 1991). Joan-F. CABESTANY I FORT, Teresa MATAS I BLANXART, ARCAT: una base de datos documental y bibliográfica para el estudio del patrimonio del arte románico catalán, "Boletin informativo del Instituto del Andaluz del Patrimonio Histórico", 7 (1994), pp. 39-40.

2"SN/G Report on Data Processing Projects in Art. Projects", I (1988), pp. 89 a 91. "SN/G Report on data Processing Projects in Art. Indexes", I (1988), p. 1.

${ }^{3}$ El seminario sobre Metodologia i problemàtiques de classificació d'una obra d'art per sistemes informàtics, tuvo lugar en la sede del Institut d'Estudis Catalans del 22 al 24 de octubre de 1990.
} 
El Centre d'Art Romànic Català (ARCAT) participó, asímismo, en la preconferencia de Bibliotecas de Arte que tuvo lugar en Barcelona del 18 al 21 de agosto de 1993, justo antes del 59 Consejo y Conferenia General de la IFLA, donde participó con una ponencia y una demostración práctica de las posiblilidades del programa ${ }^{4}$. Como consecuencia de la trayectoria y difusión alcanzada por ARCAT, a lo largo de los más de diez años de actividad, este ha recibido numerosas consultas y solicitudes de conexión nacionales y internacionales. Entre sus actividades científicas podemos destacar la oganización de VII Mesas Redondas agrupadas bajo el título genérico de Problemes $i$ Suggeriments a la recerca $i$ estudis de l'art i la història del romànic català, en las cuales se ha analizado y debatido, por prestigiosos historiadores del art y de la cultura medieval, interesantes aspectos de los diversos temas relacionados con el periodo y estilo artístico del cual se ocupa ARCAT ${ }^{5}$.

\section{OBJETIVOS}

Este programa informático tiene como principal objetivo la formación de una importante base de datos de arte románico catalan de los siglos IX al XIII, que aglutine todos los aspectos relacionados con la investigación de la arquitectura y las demás manifestaciones artísticas dependientes de esta. Otra finalidad es el análisis y estudio particular de cada uno de los monumentos inventariados.

${ }^{4}$ Joan-F. CABESTANY I Fort, M. Teresa MATAS I BLANXART, ARCAT: Una base de datos documental y bibliográfica para el estudio del patrimonio de arte románico catalán. "IFLA. Bibliotecas de arte, arquitectura y diseño: perspectivas actuales", 74 (1995), pp. 277-284.

${ }^{5}$ Las Mesas Redondas organizadas por ARCAT hasta la actualidad son:

-La "Civitas" pre-romànica d'Olèrdola. Entre Roma i el Romànic (20 de enero de 1993).

-Les tribunes: Serrabona, Cuixà $i$ Sant Llorenç prop Bagà (17 de marzo de 1993).

-Sant Vicenç d'Espinelves: Història, arquitectura i decoració (12 de mayo de 1993).

-Confessio-Cripta: Una qüestió a debatre (2 de marzo de 1994). Este acto originó la publicación de sus conclusiones en la revista "Lambard. Estudis d'art medieval", vol. VII (1995), pp. 187-211.

-Els campanars de torre amb bandes llombardes als bisbats de Vic $i$ de Girona (13 de abril de 1994).

-El claustre, àmbit monacal o espai sumptuós (29 de marzo de 1995)

-Les esglésies parroquials $i$ l'orientació de les seves portes (segles XI-XIII) (13 de noviembre de 1996). 
La metodologia utilizada en la confección del software se ajustó a la normativa internacional, con el fin de permitir establecer intercambios entre diversos centros dedicados a la realización de trabajos de investigación de caracter similar.

El Centre d'Art Romànic Català (ARCAT) pretende servir como instrumento para proporcionar una amplia y válida información que faculte la confección no sólo de trabajos de tipo general, sinó también de investigaciones de carácter más especializado como pueden ser tesis de licenciatura o tesis doctorales, entre otros numerosos estudios de diversa índole ${ }^{6}$.

Vista la complejidad del análisis del arte románico catalán, ARCAT, optó por dividir el objeto a investigar en tres grandes categorías:

a) Edificios civiles.

b) Edificios militares.

c) Edificios religiosos.

A partir de este planteamiento, se tomó, como punto de partida inicial, el estudio de los edificios de tipo religioso. Esta opción se justifica por el mayor número de construcciones de carácter religioso conservados y por las tipologías más concretos que se derivan de ellos, en comparación a las otras dos categorías de monumentos. Se tuvo también en cuenta que los edificios de tipo religioso disponen de una extensa bibliografía sobre la cual basar el contenido del programa informático. Dentro de este grupo de obras básicas cabe destacar, entre los muchos eruditos dedicados al análisis del arte románico catalán, la insigne obra de Josep Puig i Cadafalch titulada L'arquitectura romànica a Catalunya y publicada en cuatro volúmenes por el Institut d'Estudis Catalans ${ }^{7}$. Este arquitecto-historiador destaca por el caracter pionero de su obra, i por el impulso que durante las primeras décadas del siglo XX dió al estudio del arte románico catalán como miembro fundador de la Secció Històrico-Arqueològica (1907) y a través de su cargo de presidente del Institut d'Estudis Catalans ocupado entre los años 1942 y

${ }^{6} \mathrm{M}$. Teresa Matas I BlanXART, E. PiQuer FerRer, Aproximació a l'estudi tipològic dels campanars de torre de tipus llombard a la plana de Vic, "Lambard. Estudis d'Art Medieval", IV (1990), pp. 95-122. M. Teresa MATAS I BlanXART, M. Lluïsa RodríGUEZ MuÑoz, Santa Maria de Colomers: un unicum dintre de l'escultura romànica catalana, "Lambard. Estudis d'Art Medieval", V (1992), pp. 113-126. Joan-F. CABESTANY I FORT, M. Teresa MATAS I BLANXART, Aproximació a les tipologies constructives dels campanars de torre romànica a Catalunya (ss. XI-XIII), "Les Cahiers de Saint-Michel de Cuxa", XXVII (1996), pp. 25-32.

${ }^{7}$ Josep Puig y Cadafalch. Antoni Falguera Y Sivilla. Josep Goday Y CaSals, L'arquitectura romànica a Catalunya, I, II, III-A, III-B, Barcelona, 1983 (1a edición 1909, 1911, 1918). 
1956. Fue siguiendo la trayectoria de Josep Puig i Cadafalch que el Institut d'Estudis Catalans se planteó continuar y completar su labor investigadora a través del programa ARCAT.

\section{ESTRUCTURA DE LA BASE DE DATOS ARCAT}

El contenido del programa informático ARCAT se ha estructurado en tres niveles de información esencial para cada uno de los monumentos que configuran el corpus de edificios de tipo religioso del periodo románico catalán, quedando dividida esta en tres grupos:

\section{Ficha técnica:}

En este apartado se ofrecen de manera puntual todos aquellos datos referentes a la identificación de una iglesia.

Ejemplo:

$\begin{array}{ll}\text { Nom: } & \text { MONTBUI, Santa Maria } \\ \text { Renom: } & \text { LA MARE DE DÉU DE GRÀCIA/ERMITA DE LA TOSSA } \\ \text { Municipi: } & \text { SANTA MARGARIDA DE MONTBUI } \\ \text { Agregat: } & \text { AO Anoia } \\ \text { Comarca: } & \text { Vic } \\ \text { Bisbat: } & \text { Entre l'any } 1000 \text { i l'any } 1099 \\ \text { Època: } & \text { Dia_ Mes_ Any } 1035 \\ \text { Consagració: } & 41^{\circ} 33^{\prime} 20^{\prime \prime} \\ \text { Latitud: } & 1^{\circ} 34^{\prime} 49 \\ \text { Longitud: } & 3754575 \\ \text { UTM: } & \end{array}$

2. Grupos de documentación:

En estos apartados se recogen una serie de aspectos referentes a la historiografía, la documentación y otras cuestiones de tipo más puntual como es, por ejemplo, el material gráfico, bibliográfico o las dimensiones de los edificios.

Estos grupos son actualmente 39: 
-Observaciones a la cronología: Recoge los datos cronológicos de un edificio durante un periodo de construcción y la historiografía anterior del lugar.

-Observaciones a la división eclesiástica: Explica los diferentes cambios que han podido surgir en los obispados a lo largo de los siglos XII al XX.

-Observaciones a la funcionalidad del edificio religioso: Comenta las diversas categorías administrativas y jurídicas del edificio.

-Actas de consagración: Figuran los autores que hacen referencia, y se transcribe la acta si se ha encontrado.

-Observaciones a las actas de consagración: Incluye las diferentes problemáticas de datación.

-Observaciones a la toponímia: Comentario de los diferentes nombres por los cuales se conoce el edificio.

-Observaciones a las coordenadas: Da información de los diferentes sistemas de coordenadas geográficas utilizadas en la cartografía española.

-Observaciones a las comarcas: Relaciona los cambios de límites político-administrativos que se hayan podido dar en la atribución geográfica de las comarcas.

-Observaciones a los municipios: Recoge la fusión moderna de municipios a causa de la despoblación.

-Observaciones a la tipología: Hace refencia a posibles problemas planteados en la arquitectura de un monumento.

-Materiales de construcción: Describe los diferentes tipos de materiales utilizados en la edificación y elavoración de cada edificio y los objetos que este contiene.

-Planimetría: Figuran los estudios que ofrecen plantas, alzados laterales y axonometrías de las construcciones.

-Observaciones a la planimetría: Refleja las posibles contradicciones encontradas en la planimetría aportada por los diferentes autores.

-Observaciones a la orientación: Confronta opiniones contradictorias respecto a la orientación del edificio, expuestas por los diversos estudiosos.

-Dimensiones: Da medidas reales de los monumentos.

-Observaciones a las dimensiones: Señala las diferencias entre los diversos autores referentes a las medidas de los edificios.

-Tipología de los paramentos: Comentario de las diferentes técnicas constructivas. 
-Comparaciones por edificios: Reúne los que presentan paralelismos en un determinado aspecto.

-Comparaciones por elementos: Nombra los elementos comparados en el apartado anterior, situándolos en los lugares correspondientes.

-Modificaciones del edificio: Figuran los cambios de estructura que ha sufrido en época posterior a su construcción.

-Añadidos al edificio: Se relacionan las construcciones que se han agregado posteriormente a su construcción.

-Reconstrucciones y restauraciones: Explica los cambios que ha sufrido una vez considerado monumento histórico-artístico.

-Excavaciones: Figuran cronológicamente las diferentes campañas arqueológicas.

-Descubrimientos: Se relacionan cronológicamente los descubrimientos.

-Dependencias hacia otra iglesia: Describe las iglesias de las que ha sido dependiente el edificio actual.

-Iglesias dependientes: Hace referencia a las iglesias sometidas en algún momento al monumento a estudiar.

-Observaciones al estado actual: Resume su apariencia actual.

-Descripciones anteriores a la época actual: Recoge datos hoy desaparecidos.

-Observaciones a la advocación: Comenta las diferentes dedicaciones de un edificio a lo largo de su historia.

-Objetos actualmente conservados en la iglesia: Relaciona las piezas artísticas que hoy se encuentran en el interior del monumento.

-Objetos procedentes del monumento y conservados en otros lugares: Nombra los tipos de obras artísticas, indicando los lugares donde se conservan.

-Noticias de objetos actualmente perdidos: Relación de los objetos artísticos constatados por fuentes documentales y bibliográficas.

-Noticias de elementos decorativos: Describe los elementos esculturados o pintados conservados en el conjunto del edificio.

-Documentación: Aporta noticias procedentes de fuentes documentales.

-Observaciones a la documentación: Comenta, si es necesario, los diferentes criterios de los autores respecto a los documentos.

-Inscripciones: Relación y transcripción por orden cronológico de todas aquellas que figuran en un edificio. 
-Heráldica: Relación e identificación de los escudos que aparecen en un edificio.

-Material gráfico: Da información de la bibliografía que dispone de reproducciones gráficas del edificio.

-Bibliografía: Únicamente referida al monumento.

\section{Variables:}

Entendemos por variable cada una de las posibles características que configuran la definición de los elementos que constituyen un edificio. Actualmente, y como consecuencia del estudio de los 2.730 monumentos introducidos en la base de datos, disponemos de un total de 6.500 variables de análisis. Este archivo esta sujeto a un continuo incremento debido a la sucesiva incorporación de características observadas por la introducción de nuevos edificios relgiosos.

La información que figura en cada uno de los monumentos analizados procede, en su totalidad, de cuatro fuentes de información:

-Bibliográfica.

-Documental.

-Material gráfico.

-Trabajo de campo.

Una vez recopilados los datos procedentes de la bibliografía y de transformar y adaptar esta información a las posibilidades que ofrecen los sistemas informáticos, se nos plantean importantes dificultades de realización. Con frecuencia, estas se deben a que las descripciones de los autores no siempre son exhaustivas, en algunos casos son contradictorias y a veces se dan disociaciones entre la opinión de los autores y las características reales del edificio.

A menudo sucede que cuando un edificio tiene un elemento o una parte del conjunto arquitectónico muy realzada, como por ejemplo un claustro profusamente decorado, o una puerta ricamente esculpida, los historiadores centran su interés en la descripción de estas zonas, y se olvidan, con frecuencia, del resto de las características intrínsecas del monumento. Es decir, del comentario de las tipologías de ventanas, bóvedas y elementos sustentantes, por citar algunos entre muchos otros. Otra falta de información reside en el hecho de que se presuponen ciertos datos que, en algunos casos, no se ajustan a la relidad. 
En otros casos, la problemática surge de la diversidad de nomenclatura que los autores utilizan para definir las características constructivas de una parte o elemento concreto de la edificación románica catalana. Como consecuencia de ello el equipo de investigadores del programa ARCAT ha tenido que disponer de los conceptos relativos al arte y especialmente a la arquitectura románica, de manera muy concisa y concreta. Esta tarea no resulta siempre fácil ya que se deben unificar las aportaciones de los diversos autores, llegando así a poder establecer la terminología adecuada, planteada como uno de los objetivos iniciales de este a análisis.

La experiencia acumulada, nos ha demostrado que, para completar, comprobar y ampliar el exámen de un edificio, es absolutamente necesario observarlo y estudiarlo detenidamente in situ. Sin embargo, la visualización directa del monumento que, en la mayoria de las ocasiones nos permite sin problemas un estudio amplio y completo, puede ser obstaculizada, en algunos casos, por las modificaciones y restauraciones que este ha sufrido a lo largo de su historia, los cuales nos dificultan el estudio correcto de una o de la totalidad de sus partes.

Teniendo en cuenta toda esta problemática, creimos oportuno adoptar el criteric de no introducir a la base de datos ARCAT ninguna característica del edificio que no haya sido constatada previamente. Esta comprobación se realiza bien a nivel bibliográfico $o$, en su defecto, analizando dicho elemento, visualmente, mediante una labor de campo. En el caso de no haber observado correctamente una parte del edificio, queda debidamente indicado en el programa con unos signos convencionales ${ }^{8}$.

\section{SISTEMAS DE CONSULTA}

El Centre d'Art Romànic Catalá (ARCAT) tiene prevista la consulta de todos aquellos niveles de información que procedan tanto de los datos que constan en la ficha técnica, como de los grupos de documentación y del

\footnotetext{
${ }^{8}$ Seran indicados con un asterisco $(*)$ colocado a continuación del nombre de la iglesia, aquellos edificios que sólo han sido objeto de análisis in situ por su parte exterior. Se marcarán con doble asterisco $\left(^{* *}\right)$ aquellos otros cuyas características hayan sido observadas interior $y$ exteriormente. Por el contrario, los monumentos no visitados, no contienen ninguna indicación de este tipo, ya que la información que recogen es únicamente de carácter bibliográfico.
} 
conjunto de variables que analizan las características arquitectónicas y ornamentales de un edificio, a tres niveles.

El más sencillo de estos es la obtención de listados que permitan la relación de edificios introducidos a la base de datos por:

-Orden alfabético.

-Orden de código.

-Orden de municipios.

Por ejemplo, si deseamos consultar los monumentos románicos pertenecientes al municipio de Vic (Osona), el resultado conseguido es:

Municipio
VIC
VIC
VIC
VIC
VIC
VIC
VIC
VIC

Nombre del edificio

Codigo

VIC, Sant Pere

SANT SIXT DE MIRALPLÀ

47

SANT LLORENÇ DE MIRALPLÀ

73

SANT MARTÍ DE SENTFORES

SANT JAUME DELS MALATS 1231

VIC, Sant Sadurní 1232

VIC, Santa Maria $\quad 2529$

VIC, Sant Miquel 2636

Una vez obtenida esta selección, podemos consultar de cada uno de los edificios clasificados, toda la información de que dispone la base de datos. Esta misma particularidad se obtendria si la selección se hubiera efectuado por orden de código o por orden alfabético del nombre del edificio.

Más compleja resulta la consulta con la posibilidad de poder seleccionar y de combinar cada una de las variables con los grupos de documentación, las comarcas y los siglos de construcción correspondientes a cada uno de los edificios.

Estas selecciones y combinaciones pueden ser efectuadas:

a) Por comarcas.

b) Por períodos de construcción.

c) Por grupos de documentación.

d) Por variables.

Y por cualquiera de las posibles combinaciones entre ellas.

Ejemplos de selecciones: 


\section{Consulta $\mathbf{n}^{0} 1$ :}

Edificios de los siglos X al XIII que contienen inscripciones alusivas a su historia, tanto en el interior como en el exterior del monumento.

Resultado:

Nombre del edificio

Codigo

TAÜLL, Sant Climent

SANT BENET DE BAGES 18

ELNA, Santa Eulàlia i Santa Júlia 19

SANT MARTÍ DEL CANIGÓ

ARLES, Santa Maria $\quad 32$

SANTA MARIA DE MEIÀ

SANT TOMÀS DE RIUDEPERES

GIRONA, Sant Daniel $\quad 60$

SANT JULIÀ DE VILATORTA 100

VILABERTRAN, Santa Maria $\quad 234$

SANT GENÍS DE FONTANES

SANT PAU DEL CAMP 493

SANT PERE CERCADA $\quad 494$

SANT SADURNÍ DEL PLA

SANT PERE DE GALLIGANS $\quad 614$

SANT PERE DE LES PUEL-LES

SANTA MARIA D'ALAÓ $\quad 632$

SANT PERE DE RODES $\quad 645$

SANT CUGAT DEL VALLÈS $\quad 646$

SANT PERE DE CLARÀ

MONTMAGASTRE, Sant Miquel 1136

LA GARRIGA, Santa Maria del Camí 1255

SANT PERE DE VILAMAJOR 1289

SANT MARTÍ D'EMPÚRIES $\quad 2174$

SANT ESTEVE DE PERATALLADA 2602

\section{Consulta $n^{0}$ 2:}

Visualizar los edificios construidos durante los siglos XI y XII que contengan criptas.

Resultado:

Nombre del edificio

Código

CARDONA, Sant Vicenç 
$\begin{array}{ll}\text { SANT BENET DE BAGES } & 18\end{array}$

ELNA, Santa Eulàlia i Santa Júlia $\quad 19$

SANT MIQUEL DE CUIXÀ 21

SANT MARTÍ DEL CANIGÓ $\quad 22$

RODA D'ISÀVENA O DE RIBAGORÇA, Sant Vicenç 33

VIC, Sant Pere $\quad 47$

SERRATEIX, Santa Maria $\quad 48$

GRANOLLERS DE LA PLANA, Sant Esteve $\quad 86$

OLIUS, Sant Esteve 103

TREDÒS, Santa Maria de Cap d'Aran $\quad 362$

LA PARRÒQUIA D' HORTÓ, Sant Pere 409

CELLERS, Santa Maria $\quad 497$

MADRONA, Sant Pere $\quad 553$

SANT PERE DEL MONT $\quad 570$

SANTA MARIA D'ALAÓ $\quad 632$

SANT PERE DE RODES $\quad 645$

ÀGER, Sant Pere $\quad 558$

CALAFELL, Santa Creu del Castell $\quad 758$

SANT MIQUEL D'ESCORNALBOU $\quad 841$

ORISTÀ, Sant Andreu 1200

CARDET, Santa Maria $\quad 1501$

MOROR, Sant Miquel 1544

ORCAU, Mare de Déu de la Pietat 1545

LLESUI, Sant Pere 1625

SANT ESTEVE DEL MALL, Mare de Déu del Tossal 1943

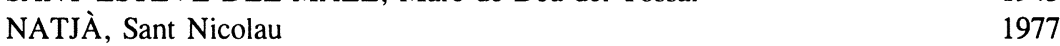

\section{Consulta $\mathbf{n}^{0}$ 3:}

Edificios de planta circular.

Resultado:

Nombre del edificio

Código

LA POBLA DE LILLET, Sant Miquel

111

SANT VICENÇ DEL CASTELL DE LLUÇÀ 112

SANT PERE EL GROS $\quad 113$

$\begin{array}{ll}\text { SANT ADJUTORI } & 114\end{array}$

$\begin{array}{ll}\text { SANT SEBASTIÀ DEL SULL } & 128\end{array}$

LA PLANA RODONA, El Sant Sepulcre 266

SALLENT DE LLOBREGAT, Sant Esteve del Castell $\quad 590$

SANT JAUME DE VILANOVA DEL PLA $\quad 602$

$\begin{array}{ll}\text { TORREBLANCA, Santa Cecília } & 1178\end{array}$ 


\section{Consulta no 4:}

Edificios de la comarca de la Conca de Barberà que sean iglesias situadas en el interior del recinto del castillo, cuya planta sea de cruz latina y que dispongan de un campanario de espadaña.

Resultado:

Nombre del edificio

BIURE DE GAIÀ, Sant Miquel de Montclar $\quad 907$

EL FONOLL, Sant Blai $\quad 918$

FORÈS, Sant Miquel $\quad 919$

MONTARGULL, Sant Jaume $\quad 921$

SABELLÀ, Sant Pere $\quad 927$

SANTA PERPÈTUA DE GAIÀ, Santa Maria $\quad 928$

GUIALMONS, Mare de Déu del Roser $\quad 2481$

SANT MIQUEL DE LA PORTELLA

$\begin{array}{ll}\text { PONTILS, Santa Maria } & 2518\end{array}$

El programa ARCAT permite, asímismo, plantear las consultas por exclusión o inclusión, y continuar la selección de un grupo de variables a partir del resultado de una primera selección hasta llegar al análisis concreto del elemento objeto de estudio o de interés.

Ejemplo de combinatoria de selecciones:

Visualizar todas las iglesias que posean alguna puerta correspondiente a la tipología de arcos degradados con arquivoltas.

Resultado: 124 edificios 9 .

Obtenida esta selección podemos continuar solicitando características más particulares, como por ejemplo: visualizar cuales tienen dos arquivoltas decoradas.

Resultado:

Nombre del edificio

Código

CORNELLÀ DE CONFLENT, Santa Maria 23

ORGANYÀ, Santa Maria $\quad 35$

CISTELLA, Santa Maria

${ }^{9} \mathrm{El}$ listado de los edificios que cumplen las características solicitadas no se especifica aquí debido a que, por su larga extensión, podria entorpecer la lectura del presente artículo. 
LLEDÓ D'EMPORDÀ, Santa Maria $\quad 176$

MAÇANET DE CABRENYS, Sant Martí $\quad 179$

COVET, Santa Maria $\quad 307$

GUILS DE CERDANYA, Sant Esteve 311

$\begin{array}{ll}\text { BEGET, Sant Cristòfol } & 617\end{array}$

PUIG-REIG, Sant Martí $\quad 630$

De este grupo de edificios se pueden seleccionar todavía, por ejemplo, aquellos que tienen capiteles decorados.

Resultado:

Nombre del edificio

Código

CORNELLÀ DE CONFLENT, Santa Maria

ORGANYÀ, Santa Maria $\quad 35$

CISTELLA, Santa Maria $\quad 153$

LLEDÓ D'EMPORDÀ, Santa Maria $\quad 176$

$\begin{array}{ll}\text { COVET, Santa Maria } & 307\end{array}$

GUILS DE CERDANYA, Sant Esteve $\quad 311$

$\begin{array}{ll}\text { BEGET, Sant Cristòfol } & 617\end{array}$

PUIG-REIG, Sant Martí $\quad 630$

Y de estos cuales tienen, además, las bases ornamentadas.

Resultado:

Nombre del edificio

Código

ORGANYÀ, Santa Maria $\quad 35$

CISTELLA, Santa Maria 153

\section{PROTOCOLOS DE CONEXIÓN Y CONSULTA}

Actualmente, esta base de datos puede ser consultada via internet previa solicitud a los directores del programa ARCAT y al presidente del Institut d'Estudis Catalans, Dr. Manuel Castellet, indicando la universidad, centro de investigación o entidad a la cual pertenece y la finalidad de su conexión. Esta petición puede cursarse por correo ordinario a:

Centre d'Art Romànic Català (ARCAT)

C/ Carme, 47. 
08001-Barcelona

O por correo electrónico o fax:

Air-Mail: ARCAT @ IEC. ES

Fax: (93) 412-29-94

Para obtener cualquier aclaración o mayor información sobre la base de datos ARCAT pueden ponerse en contacto telefónico a través de los números: 318-55-50, 318-55-16 (ext. 259).

\section{RÉSUMÉ}

ARCAT est un programme informatique consacré à l'étude de l'art roman de la Catalogne. Cette Base de Données a une méthodologie que analyse et classe toutes les catégories stylistiques, ornamentales, picturales et des arts somptuaires présentes dans les édifices religieux. Actuellement l'inventaire dépasse le chiffre de 2730 monuments.

Le programme dispose de 6500 variants qui donnent les diverses caractéristiques architecturales de la definition du bâtiment. En outre, cette information est enrichie par 39 grupes de documentation qui couvrent l'analyse artistique de chaque monument avec una base historique, documentaire et bibliographique.

Le programme offre la possibilité de consulter chaque édifice particulièrement. Il permet également la selection conjointe des lieux geographiques, des époques de construction, des groupes de documentation et des variants. On peut aussi, continuer la selection d'un groupe de variants depuis la première recherche jusqu'à l'analyse de chaque élément d'étude proposé.

\section{SUMMARY}

The ARCAT programme with the application of an advenced methodology, especially created for the study of Romanic Art, analyses, clasifies and studies the different stylistic, ornamental, pictoric, sumptuary and minor art categories of religious building which now excced 2730 in number, checked in the data bank.

The Program has available 6500 variables wich take into account the defining architectural characteristics of a building. This information also includes 39 groups of documentation which completes the artistic analysis of each monument with an historical, documental and bibliographical base.

The Program lest anything about a concrete building to be consulted. In the same way, it allows the whole selection of geographical places, construction periods, units of documentation and variables.

It is also possible to select a group of variables from a first choice to reach the concrete analysis of an element. 